\title{
Global Periodic Solutions in a Delayed Predator-Prey System with Holling II Functional Response
}

\author{
ZHICHAO JIANG* \\ Fundamental Science Department, North China Institute of Astronautic Engineer- \\ ing, Hebei, Langfang, 065000, China \\ e-mail : jiangzhichao1980@163.com \\ HONGTAO WANG \\ Heibei College of Finance, Hebei, Baoding, 071051, China \\ e-mail : wanghongtao05@gmail.com \\ HONGMEI WANG \\ North China Electric Power University, Hebei, Baoding, 071051, China \\ e-mail : red-amei81110@sina.com
}

\begin{abstract}
We consider a delayed predator-prey system with Holling II functional response. Firstly, the paper considers the stability and local Hopf bifurcation for a delayed prey-predator model using the basic theorem on zeros of general transcendental function, which was established by Cook etc.. Secondly, special attention is paid to the global existence of periodic solutions bifurcating from Hopf bifurcations. By using a global Hopf bifurcation result due to $\mathrm{Wu}$, we show that the local Hopf bifurcation implies the global Hopf bifurcation after the second critical value of delay. Finally, several numerical simulations supporting the theoretical analysis are given.
\end{abstract}

\section{Introduction}

For a long time, the global existence of periodic solutions to the mathematical models of population dynamics has attracted much attention due to its theoretical and practical significance. It is well known that periodic solutions can arise through the Hopf bifurcation in delay differential equations. However, these periodic solutions bifurcating from Hopf bifurcations are generally local. Therefore, it is an important mathematical subject to investigate if these non-constant periodic solutions which are obtained through local Hopf bifurcations exist globally. Recently, a great deal of research has been devoted to the topics. One of the methods used in them is the ejective fixed point argument developed by Nussbaum [1], which has been successfully used to obtain the global existence of periodic solutions bifurcat-

\footnotetext{
* Corresponding author.

Received June 16, 2009; accepted January 27, 2010.

2000 Mathematics Subject Classification: 34K18, 34K20, 92B20.

Key words and phrases: Time delay; Hopf bifurcation; Numerical simulations.
} 
ing from the Hopf bifurcation by many researchers (see, e.g., [2-5]). The other is the global Hopf bifurcation theorem due to Erbe et al. [6], which was established using a purely topological argument. Krawcewicz et al. [7] firstly applied this global Hopf bifurcation theorem to a neutral functional differential equation. Thereafter, many researchers have employed it to investigate the global existence of periodic solutions for retarded functional differential equations (see, e.g., [8-15]). We would like to mention that, several papers have studied stability of functional equations( see, e.g., [16-18]).

In the present paper, we again devote our attention to the global existence of periodic solutions to the following predator-prey system:

$$
\left\{\begin{array}{l}
\dot{x}(t)=x(t)\left[r_{1}-a_{11} x(t-\tau)-\frac{a_{12} y(t)}{1+m x(t)}\right] \\
\dot{y}(t)=y(t)\left[-r_{2}+\frac{a_{21} x(t)}{1+m x(t)}-a_{22} y(t)\right],
\end{array}\right.
$$

where $x(t)$ and $y(t)$ denote the density of prey and predator at time $t$, respectively. $\mathrm{m}$ denotes the search rate multiplied by the handling time; $r_{1}$ denotes the intrinsic growth rate of prey; $r_{2}$ denotes the death rate of the predator; $a_{11}$ denotes the intraspecific competitions rate of the prey; $a_{12}$ denotes the capturing rate of the predator; $\frac{a_{21}}{a_{12}}$ is the conversion rate of nutrients into the reproduction of the predator; $a_{22}$ is the interspecies competitions rate of the prey and predator; $\tau$ is the generation time of the prey species. In biological terms, $\tau, r_{i}, a_{i j}(i, j=1,2)$ are positive constants. The main purpose of this paper is to show that the local Hopf bifurcation of system (1.1) implies the global Hopf bifurcation after the second critical value of delay, by using a global Hopf bifurcation theorem in $\mathrm{Wu}$ [15].

This paper is organized as follows. In the next section, we shall consider the stability and the local Hopf bifurcation of the positive equilibrium. In Section 3, the global existence of these bifurcating periodic solutions will be considered. We shall give some numerical simulations in Section 4.

\section{Stability of the positive equilibrium and local Hopf bifurcations}

It is obvious that system (1.1) has three boundary equilibria: $E_{1}(0,0), E_{2}\left(0,-\frac{r_{2}}{a_{22}}\right)$, $E_{3}\left(\frac{r_{1}}{a_{11}}, 0\right)$.

(P1) $r_{1} a_{12}-r_{2} a_{11}-m r_{1} r_{2}>0$ and $m r_{1}<a_{11}$.

Proposition 2.1. If the condition ( $P 1)$ holds, then system (1.1) has a unique positive equilibrium $E^{*}\left(x^{*}, y^{*}\right)$, where $x^{*} \in\left(0, \frac{r_{1}}{a_{11}}\right)$.

In fact, from the second equation of system (1.1), we have

$$
y=\frac{1}{a_{22}}\left[-r_{2}+\frac{a_{21} x}{1+m x}\right] .
$$

Let

$$
H(x)=r_{1}-a_{11} x+\frac{a_{12} r_{2}}{a_{22}(1+m x)}-\frac{a_{12} a_{21} x}{a_{22}(1+m x)^{2}} .
$$


Hence we get that

$$
\lim _{x \rightarrow 0} H(x)=r_{1}+\frac{a_{12} r_{2}}{a_{22}}>0
$$

and

$$
\lim _{x \rightarrow \frac{r_{1}}{a_{11}}} H(x)=\frac{a_{12} a_{11}^{2}\left[a_{11} r_{2}-a_{12} r_{1}+m r_{1} r_{2}\right]}{a_{11} a_{22}\left(a_{11}+m r_{1}\right)^{2}} .
$$

Furthermore, we have that $H^{\prime}(x)=-a_{11}-\frac{a_{12} m r_{2}}{a_{22}(1+m x)^{2}}-\frac{a_{12} a_{21}(1-m x)}{a_{22}(1+m x)^{3}}$. Unde the condition (P1), we get that $H^{\prime}(x)<0$ and $\lim _{x \rightarrow \frac{r_{1}}{a_{11}}} H(x)<0$. Hence system (1.1) has a unique positive equilibrium $E^{*}\left(x^{*}, y^{*}\right)$ under the condition (P1), where $x^{*} \in\left(0, \frac{r_{1}}{a_{11}}\right)$.

By the translation $u_{1}(t)=x(t)-x^{*}, u_{2}(t)=y(t)-y^{*}$, system (1.1) is written as

$$
\left\{\begin{array}{l}
\dot{u}_{1}(t)=\left[u_{1}(t)+x^{*}\right]\left[r_{1}-a_{11}\left(u_{1}(t-\tau)+x^{*}\right)-\frac{a_{12}\left(u_{2}(t)+y^{*}\right)}{1+m\left(u_{1}(t)+x^{*}\right)}\right] \\
\dot{u}_{2}(t)=\left[u_{2}(t)+y^{*}\right]\left[-r_{2}+\frac{a_{21}\left(u_{1}(t) *\right)}{1+m\left(u_{1}(t)+x^{*}\right)}-a_{22}\left(u_{2}(t)+y^{*}\right)\right] .
\end{array}\right.
$$

The linearization of Eq. $(2.3)$ at $(0,0)$ is

$$
\left\{\begin{array}{l}
\dot{u}_{1}(t)=\frac{m a_{12} x^{*} y^{*}}{\left(1+m x^{*}\right)^{2}} u_{1}(t)-a_{11} x^{*} u_{1}(t-\tau)-\frac{a_{12} x^{*}}{1+m x^{*}} u_{2}(t) \\
\dot{u}_{2}(t)=\frac{a_{21} y^{*}}{\left(1+m x^{*}\right)^{2}}-a_{22} y^{*} u_{2}(t),
\end{array}\right.
$$

whose characteristic equation is

$$
\lambda^{2}+p \lambda+r+(s \lambda+q) e^{-\lambda \tau}=0,
$$

where

$$
\begin{gathered}
p=a_{22} y^{*}-\frac{m a_{12} x^{*} y^{*}}{\left(1+m x^{*}\right)^{2}}, q=a_{11} a_{22} x^{*} y^{*}, \\
r=\frac{a_{12} x^{*} y^{*}\left[a_{21}-m a_{22} y^{*}-m^{2} a_{22} x^{*} y^{*}\right]}{\left(1+m x^{*}\right)^{3}}, s=a_{11} x^{*} .
\end{gathered}
$$

The second-degree transcendental polynomial equation (2.5) has been extensively studied by many researchers (see, e.g., [19-20]). In particular, we introduce the following results stated in Ruan [19] about the distributions of the roots of the characteristic Eq. (2.5).

Let

$(P 2) p+s>0$

$(P 3) q+r>0$

$(P 4)$ either $s^{2}-p^{2}+2 r<0$ and $r^{2}-q^{2}>0$ or $\left(s^{2}-p^{2}+2 r\right)^{2}<4\left(r^{2}-q^{2}\right)$;

$(P 5) r^{2}-q^{2}<0$ or $s^{2}-p^{2}+2 r>0$ and $\left(s^{2}-p^{2}+2 r\right)^{2}=4\left(r^{2}-q^{2}\right)$;

(P6) $r^{2}-q^{2}>0, s^{2}-p^{2}+2 r>0$ and $\left(s^{2}-p^{2}+2 r\right)^{2}>4\left(r^{2}-q^{2}\right)$. 
Lemma 2.1([19]). For Eq.(2.5), we have

(i) If (P2), (P3) and (P4) hold, then all roots of Eq.(2.5) have negative real parts for all $\tau \geq 0$.

(ii) If (P2), (P3), and (P5) hold and $\tau=\tau_{j}^{+}$, then Eq. (2.5) has a pair of purely imaginary roots $\pm i \omega_{+}$. When $\tau=\tau_{0}^{+}$, then all roots of Eq. (2.5) except $\pm \omega_{+} i$ have negative real parts.

(iii) If (P2), (P3), and (P6) hold and $\tau=\tau_{j}^{+}\left(\tau=\tau_{j}^{-}\right.$, respectively), then Eq. (2.5) has a pair of imaginary roots $\pm i \omega_{+}\left( \pm i \omega_{-}\right.$, respectively). Furthermore, when $\tau=\tau_{j}^{+}\left(\tau=\tau_{j}^{-}\right.$, respectively $)$, then all roots of Eq. (2.5) except $\pm i \omega_{+}\left( \pm i \omega_{-}\right.$, respectively) have negative real parts.

Here,

$$
\left.\omega_{ \pm}=\sqrt{\frac{\sqrt{2}}{2}\left\{s^{2}-p^{2}+2 r \pm \sqrt{\left(s^{2}-p^{2}+2 r\right)^{2}-4\left(r^{2}-q^{2}\right)}\right.}\right\}
$$

and

$$
\tau_{j} \pm=\frac{1}{\omega_{ \pm}} \arccos \left[\frac{q\left(\omega_{ \pm}^{2}-r\right)-p s \omega_{ \pm}^{2}}{s^{2} \omega_{ \pm}^{2}+q^{2}}\right]+\frac{2 j \pi}{\omega_{ \pm}}, j=0,1,2, \cdots
$$

From Lemma 2.1, we easily obtain the following results about the stability and the Hopf bifurcation of system (1.1) of the positive equilibrium $E^{*}$.

Theorem 2.1. Suppose that $p+s>0$ and $q+r>0$ are satisfied. From system (1.1), we have

(i) if either $s^{2}-p^{2}+2 r<0$ and $r^{2}-q^{2}>0$ or $\left(s^{2}-p^{2}+2 r\right)^{2}<4\left(r^{2}-q^{2}\right)$, then the equilibrium $E^{*}$ of the system (1.1) is asymptotically stable for all $\tau \geq 0$;

(ii) if $q-r>0$, then $E^{*}$ is asymptotically stable when $\tau \in\left[0, \tau_{0}^{+}\right)$and unstable when $\tau>\tau_{j}^{+}$. System (1.1) undergoes a Hopf bifurcation at $E^{*}$ when $\tau=\tau_{j}^{+}$;

(iii) if $q-r<0, s^{2}-p^{2}+2 r>0$ and $\left(s^{2}-p^{2}+2 r\right)^{2}>4\left(r^{2}-q^{2}\right)$ hold, then there is a positive integer $k$, such that the equilibrium $E^{*}$ switches $k$ times from stability to instability to stability; that is, $E^{*}$ is asymptotically stable when

and unstable when

$$
\tau \in\left[0, \tau_{0}^{+}\right) \cup\left(\tau_{0}^{-}, \tau_{1}^{+}\right) \cup \cdots \cup\left(\tau_{k-1}^{-}, \tau_{k}^{+}\right)
$$

$$
\tau \in\left[\tau_{0}^{+}, \tau_{0}^{-}\right) \cup\left[\tau_{1}^{+}, \tau_{1}^{-}\right) \cup \cdots \cup\left[\tau_{k-1}^{+}, \tau_{k-1}^{-}\right) \text {and } \tau>\tau_{k}^{+} .
$$

Here, $\omega_{ \pm}$and $\tau_{j}^{ \pm}$are defined as follows (2.6) and (2.7), respectively.

Let $\lambda_{j}=\alpha_{j}(\tau)+i \omega_{j}(\tau), j=0,1,2, \cdots$ be the root of Eq. (2.5) satisfying $\alpha_{j}\left(\tau_{j}^{ \pm}\right)=0, \omega_{j}\left(\tau_{j}^{ \pm}\right)=\omega_{ \pm}$. Then the following transversally conditions hold:

$$
\frac{d}{d \tau} \operatorname{Re} \lambda_{j}\left(\tau_{j}^{+}\right)>0, \frac{d}{d \tau} \operatorname{Re} \lambda_{j}\left(\tau_{j}^{-}\right)<0
$$




\section{Global existence of periodic solutions}

In this section, we study the global continuation of periodic solutions bifurcating from the equilibrium $E^{*}, \tau_{j}^{+}(j=1,2, \cdots)$ for system (1.1). Throughout this section, we follow closely the notations in [15]. For simplification of notations, setting $z_{t}=\left(x_{t}, y_{t}\right)$, we may rewrite systems (1.1) as the following functional differential equation:

$$
\dot{z}(t)=F\left(z_{t}, \tau, p\right),
$$

where $z_{t}(\theta)=z(t+\theta) \in C\left([-\tau, 0], R^{2}\right)$. It is obvious that system (3.1) has four equilibria $\bar{z}_{1}=(0,0), \bar{z}_{2}=\left(0,-\frac{r_{2}}{a_{22}}\right), \bar{z}_{3}=\left(\frac{r_{1}}{a_{11}}, 0\right)$ and $z^{*}=E^{*}$. Following the work of $\mathrm{Wu}[15]$, we need to define

$$
\begin{aligned}
& X=C\left([-\tau, 0], R^{2}\right) \\
& \Sigma=C L\left\{(z(t), \tau, p) \in X \times R \times R^{+} ; z(t+p)=z(t)\right\} \\
& N=\{(\bar{z}, \bar{\tau}, \bar{p}) ; F(\bar{z}, \bar{\tau}, \bar{p})=0\}
\end{aligned}
$$

and let $\ell_{\left(z^{*}, \tau_{j}^{+}, \frac{2 \pi}{\omega_{+}}\right)}$denote the connected component of $\left(z^{*}, \tau_{j}^{+}, \frac{2 \pi}{\omega_{+}}\right)$in $\Sigma$, where $\tau_{j}^{+}$ and $\omega_{+}$are defined in (2.6) and (2.7), respectively.

Lemma 3.1. If the condition $(P 1)$ holds, then all the nontrivial periodic solutions of system (1.1) are uniformly bounded.

Proof. For periodic functions $x(t)$ and $y(t)$, we define

$$
\begin{aligned}
& x\left(\xi_{1}\right)=\min \{x(t)\}, x\left(\eta_{1}\right)=\max \{x(t)\}, \\
& y\left(\xi_{2}\right)=\min \{y(t)\}, y\left(\eta_{2}\right)=\max \{y(t)\} .
\end{aligned}
$$

Let $(x(t), y(t))$ be a nonconstant periodic solution of system (1.1). Then we obtain

$$
\left\{\begin{array}{l}
x(t)=x(0) e^{\int_{0}^{t}\left[r_{1}-a_{11} x(s-\tau)-\frac{a_{12} y(s)}{1+m x(s)}\right] d s} \\
y(t)=y(0) e^{\int_{0}^{t}\left[-r_{2}+\frac{a_{12} x(s)}{1+m x(s)}-a_{22} y(s)\right] d s}
\end{array}\right.
$$

which implies either $x(t) \equiv 0$ or $x(t) \neq 0$, and either $y(t) \equiv 0$ or $y(t) \neq 0$.

(I) When $x(t)>0, y(t)>0$ (or $y(t) \equiv 0$ )from the first equation of system (1.1), we have,

$$
0=r_{1}-a_{11} x\left(\eta_{1}-\tau\right)-\frac{a_{12} y\left(\eta_{1}\right)}{1+\gamma_{1} x\left(\eta_{1}\right)} \leq r_{1}-a_{11} x\left(\eta_{1}-\tau\right)
$$

and

$$
\dot{x}(t)<r_{1} x(t)
$$

which leads to

$$
x\left(\eta_{1}-\tau\right) \leq \frac{r_{1}}{a_{11}}, \quad x(t)<e^{r_{1} \tau} x(t-\tau) .
$$


It follows from (3.2) and (3.3) that

$$
x\left(\eta_{1}\right) \leq \frac{r_{1}}{a_{11}} e^{r_{1} \tau} .
$$

On the other hand, from the second equation of system (1.1), we get,

$$
0=-r_{2}+\frac{a_{21} x\left(\eta_{2}\right)}{1+m x\left(\eta_{2}\right)-a_{22} y\left(\eta_{2}\right)}-a_{22} y\left(\eta_{2}\right) \leq-r_{2}+\frac{a_{21} r_{1} e^{r_{1} \tau}}{a_{11}+m r_{1} e^{r_{1} \tau}}-a_{22} y\left(\eta_{2}\right) .
$$

It follows that

$$
y\left(\eta_{2}\right) \leq-\frac{r_{2}}{a_{22}}+\frac{a_{21} r_{1} e^{r_{1} \tau}}{a_{22}\left(a_{11}+m r_{1} e^{r_{1} \tau}\right)} .
$$

(II) If $x(t)>0 y(t)<0$, then from the second equation of system (1.1) we obtain

$$
0=-r_{2}+\frac{a_{21} x\left(\xi_{2}\right)}{1+\gamma_{1} x\left(\xi_{2}\right)}-a_{22} y\left(\xi_{2}\right)>-r_{2}-a_{22} y\left(\xi_{2}\right),
$$

which means

$$
y\left(\xi_{2}\right)>-\frac{r_{2}}{a_{22}} .
$$

It follows together with the first equation of system (1.1) that

$$
\dot{x}(t)<x(t)\left[r_{1}-a_{11} x(t-\tau)+\frac{a_{12} r_{2}}{a_{22}}\right]<\frac{r_{1} a_{22}+r_{2} a_{12}}{a_{22}} x,
$$

which induce

$$
x(t)<x(t-\tau) e^{\left\{\frac{\left[r_{1} a_{22}+r_{2} a_{12}\right] \tau}{a_{22}}\right\}},
$$

i.e.

$$
x(t-\tau)>e^{-\left\{\frac{\left[r_{1} a_{22}+r_{2} a_{12}\right] \tau}{a_{22}}\right\} x(t) .}
$$

Thus, we obtain

$$
\dot{x}(t)<x(t)\left[\frac{r_{1} a_{22}+r_{2} a_{12}}{a_{22}}-a_{11} e^{-\left\{\frac{\left[r_{1} a_{22}+r_{2} a_{12}\right] \tau}{a_{22}}\right\} x(t)}\right] .
$$

By comparison, (3.7) implies that $x(t)$ is bounded above by the solution of

$$
\dot{u}(t)<u(t)\left[\frac{r_{1} a_{22}+r_{2} a_{12}}{a_{22}}-a_{11} e^{-\left\{\frac{\left[r_{1} a_{22}+r_{2} a_{12}\right] \tau}{a_{22}}\right\} u(t)}\right],
$$

satisfying $u(0)=x(0)$. Define $u(t)$ by the solution of (3.8) starting at $u(0)>0$. Then we have

$$
x\left(\eta_{1}\right)<\frac{\left[r_{1} a_{22}+r_{2} a_{12}\right]}{a_{11} a_{22}} e^{\left\{\frac{\left[r_{1} a_{22}+r_{2} a_{12}\right] \tau}{a_{22}}\right\}}+1 .
$$


(III) If $x(t)<0, y(t)>0$, then from the second equation of system (1.1) we obtain

$$
0=-r_{2}+\frac{a_{12} x\left(\xi_{2}\right)}{1+\gamma_{1} x\left(\xi_{2}\right)}-a_{22} y\left(\xi_{2}\right)<-r_{2}-a_{22} y\left(\xi_{2}\right),
$$

which leads to

$$
y\left(\xi_{2}\right)<-\frac{r_{2}}{a_{22}}<0 .
$$

Obviously, it is a contradiction. Thus, there are no nontrivial periodic solutions to system (1.1) in this case. Use the same methods, we can obtain that there are no nontrivial periodic solutions of system $(1.1)$ when $x(t)<0, y(t)<0$ or $y(t) \equiv 0$ and $x(t) \equiv 0, y(t) \neq 0$. Therefore, suppose that $(x(t), y(t))$ is a nonconstant periodic solution of system (1.1), then from (3.4) and (3.9) we have

$$
0<x(t) \leq \max \left\{\frac{r_{1} e^{r_{1} \tau}}{a_{11}}, \frac{\left[r_{1} a_{22}+r_{2} a_{12}\right]}{a_{11} a_{22}} e^{\left\{\frac{\left[r_{1} a_{22}+r_{2} a_{12}\right] \tau}{a_{22}}\right\}}+1\right\},
$$

and from (3.5) and (3.6) we get

$$
-\frac{r_{2}}{a_{22}}<y(t) \leq-\frac{r_{2}}{a_{22}}+\frac{a_{21} r_{1} e^{r_{1} \tau}}{a_{22}\left(a_{11}+m r_{1} e^{r_{1} \tau}\right)} .
$$

Therefore, Lemma 3.1 is true.

Lemma 3.2. When the condition (P1) holds, system (1.1) has no any nontrivial $\tau$-periodic solutions.

Proof. For a contradiction, suppose that system (1.1) has $\tau$-periodic solution. Then the following system (3.10) of ordinary differential equations has periodic solution:

$$
\left\{\begin{array}{l}
\dot{x}(t)=x(t)\left[r_{1}-a_{11} x(t)-\frac{a_{12} y(t)}{1+m x(t)}\right] \\
\dot{y}(t)=y(t)\left[-r_{2}+\frac{a_{21} x(t)}{1+m x(t)}-a_{22} y(t)\right],
\end{array}\right.
$$

which has the same equilibria to system (1.1), i.e. $\bar{z}_{1}=(0,0), \bar{z}_{2}=\left(0,-\frac{r_{2}}{a_{22}}\right)$, $\bar{z}_{3}=\left(\frac{r_{1}}{a_{11}}, 0\right)$ and a unique positive equilibrium $z^{*}=E^{*}$. Note that $x$-axis and $y$ axis are the invariable manifold of system (3.10) and the orbits of system (3.10) do not intersect each other. Thus, there are no solutions crossing the coordinate axes. On the other hand, we note the fact that if system (3.10) has a periodic solution, then there must be the equilibrium in its interior, and that $\bar{z}_{1}, \bar{z}_{2}$ and $\bar{z}_{3}$ are located on the coordinate axes. Thus, we conclude that the periodic orbit of system (3.10) must lie in the first quadrant. Using the same methods as [21-23], we have that the positive equilibrium $E^{*}$ is global asymptotically stable in the first quadrant. Thus, there is not periodic orbit in the first quadrant too. It is a contradiction. The above discussion means that (3.10) has no any nontrivial periodic solutions. Therefore, 
Lemma 3.2 is confirmed.

Theorem 3.1. Suppose that $q-r>0$ and the condition $(P 1)$ hold. Then for each $\tau>\tau_{j}^{+}(j=1,2, \cdots)$, system (1.1) has at least $k-1$ periodic solutions.

Proof. It is sufficient to prove that the projection of $\ell_{\left(z^{*}, \tau^{j}, \frac{2 \pi}{\omega_{+}}\right)}$onto $\tau$-space is $[\bar{\tau}, \infty)$ for each $j \geq 1$, where $\bar{\tau} \leq \tau_{j}^{+}$. The characteristic matrix of (3.1) at an equilibrium $\bar{z}=\left(\bar{z}^{(1)}, \bar{z}^{(2)}\right) \in R^{2}$ takes the following form:

$$
\Delta(\bar{z}, \tau, p)(\lambda)=\lambda I d-D F(\bar{z}, \bar{\tau}, \bar{p})\left(e^{\lambda \cdot} I d\right),
$$

i.e.

$$
\begin{aligned}
& \Delta(\bar{z}, \tau, p)(\lambda) \\
= & \left(\begin{array}{lc}
\lambda-r_{1}+a_{11} \bar{z}^{(1)}\left(1+e^{-\lambda \tau}\right)+\frac{a_{12} \bar{z}^{(2)}}{\left(1+m \bar{z}^{(1)}\right)^{2}} & \frac{a_{12} \bar{z}^{(1)}}{1+m \bar{z}^{(1)}} \\
-\frac{a_{21} \bar{z}^{(2)}}{\left(1+m \bar{z} \bar{z}^{(1)}\right)^{2}} & \lambda+r_{2}-\frac{a_{21} \bar{z}^{(1)}}{1+m \bar{z}^{(1)}}+2 a_{22} \bar{z}^{(2)}
\end{array}\right),
\end{aligned}
$$

$(\bar{z}, \bar{\tau}, \bar{p})$ is called a center if $F(\bar{z}, \bar{\tau}, \bar{p})=0$ and $\Delta(\bar{z}, \bar{\tau}, \bar{p})\left(\frac{2 \pi}{p} i\right)=0$. A center $(\bar{z}, \bar{\tau}, \bar{p})$ is said to be isolated if it is the only center in some neighborhood of $(\bar{z}, \bar{\tau}, \bar{p})$. It follows from (3.11) that

$$
\begin{gathered}
\operatorname{det}\left(\Delta\left(\bar{z}_{1}, \tau, p\right)(\lambda)\right)=\left(\lambda-r_{1}\right)\left(\lambda+r_{2}\right)=0, \\
\operatorname{det}\left(\Delta\left(\bar{z}_{2}, \tau, p\right)(\lambda)\right)=\left(\lambda-r_{1}-\frac{a_{12} r_{2}}{a_{22}}\right)\left(\lambda-r_{2}\right)=0, \\
\operatorname{det}\left(\Delta\left(\bar{z}_{3}, \tau, p\right)(\lambda)\right)=\left(\lambda+r_{1} e^{-\lambda \tau}\right)\left(\lambda+r_{2}-\frac{a_{21} r_{1}}{a_{11}+m r_{1}}\right)=0,
\end{gathered}
$$

Obviously, (3.12) and (3.13) have no purely imaginary roots. Thus, we conclude that (3.1) has no the center of the form as $\left(\bar{z}_{i}, \tau, p\right)(i=1,2)$. For $\omega>0, i \omega$ is a root of (3.14) if and only if

$$
i \omega+r_{1}(\cos \omega \tau-i \sin \omega \tau)=0 .
$$

Separating the real and imaginary parts, we have

$$
\left\{\begin{array}{l}
r_{1} \cos \omega \tau=0 \\
r_{1} \sin \omega \tau=\omega
\end{array}\right.
$$

which implies

$$
\left\{\begin{array}{l}
\omega=r_{1}, \\
\tau_{k}=\frac{\pi}{2 r_{1}}+\frac{2 k \pi}{r_{1}} .
\end{array}\right.
$$

Thus, when $\tau_{k}=\frac{\pi}{2 r_{1}}+\frac{2 k \pi}{r_{1}},(3.14)$ has a pair of simple imaginary roots $\pm i r_{1}$. By direction computation, we may obtain that

$$
\operatorname{Re}\left\{\left.\frac{d \lambda}{d \tau}\right|_{\tau=\tau_{k}}\right\}=\frac{r_{1}^{2}}{1+r_{1}^{2} \tau_{k}^{2}}>0
$$


Therefore, we conclude that $\left(\bar{z}_{3}, \tau_{k}, \frac{2 \pi}{r_{1}}\right)$ is a isolated center stated as above. On the other hand, from the discussion about the local Hopf bifurcation in Section 2 , it is easy to verify that $\left(z^{*}, \tau_{j}^{+}, \frac{2 \pi}{\omega_{+}}\right)$is also a isolated center, and there exist $\varepsilon>0, \delta>0$ and a smooth curve $\lambda:\left(\tau^{j}-\delta, \tau^{j}+\delta \rightarrow C\right)$ such that $\operatorname{det}(\triangle(\lambda(\tau)))=$ $0,\left|\lambda(\tau)-\omega_{+} i\right|<\varepsilon$ for all $\tau \in\left[\tau_{j}^{+}-\delta, \tau_{j}^{+}+\delta\right]$ and $\lambda\left(\tau_{j}^{+}\right)=\omega_{+} i, \frac{d \operatorname{Re} \lambda\left(\tau_{j}^{+}\right)}{d \tau}>0$.

Let $\Omega_{\epsilon, \frac{2 \pi}{\omega_{+}}}=\left\{(\eta, p): 0<\eta<\varepsilon,\left|p-\frac{2 \pi}{\omega_{+}}\right|<\varepsilon\right\}$. It is easy to verify that on $\left[\tau_{j}^{+}-\delta, \tau_{j}^{+}+\delta\right] \times \partial \Omega_{\varepsilon, \frac{2 \pi}{\omega_{+}}}, \Delta\left(z^{*}, \tau, p\right)\left(\eta+\frac{2 \pi}{p} i\right)=0$ if and only if $\eta=0, \tau=\tau_{j}^{+}, p=\frac{2 \pi}{\omega_{+}}$. Therefore, the hypotheses $(A 1)-(A 4)$ in [15] are satisfied. Moreover, if we define

$$
H^{ \pm}\left(z^{*}, \tau_{j}^{+}, \frac{2 \pi}{\omega_{+}}\right)(\eta, p)=\Delta\left(z^{*}, \tau_{j}^{+} \pm \delta, p\right)\left(\eta+\frac{2 \pi}{p} i\right),
$$

then we have the crossing number of isolated center $\left(z^{*}, \tau_{j}^{+}, \frac{2 \pi}{\omega_{+}}\right)$as follows:

$\gamma\left(z^{*}, \tau_{j}^{+}, \frac{2 \pi}{\omega_{+}}\right)=\operatorname{deg}_{B}\left(H^{-}\left(z^{*}, \tau_{j}^{+}, \frac{2 \pi}{\omega_{+}}\right), \Omega_{\epsilon, \frac{2 \pi}{\omega_{+}}}\right)-\operatorname{deg}_{B}\left(H^{+}\left(z^{*}, \tau_{j}^{+}, \frac{2 \pi}{\omega_{+}}\right), \Omega_{\epsilon, \frac{2 \pi}{\omega_{+}}}\right)=-1$.

For the isolated center $\left(\bar{z}_{3}, \tau_{k}, \frac{2 \pi}{r_{1}}\right)$, the similar arguments may also show that $\gamma\left(\bar{z}_{3}, \tau_{j}^{+}, \frac{2 \pi}{\omega_{+}}\right)=-1$. Thus we have that if $(\bar{z}, \bar{\tau}, \bar{p}) \in \ell_{\left(z^{*}, \tau_{j}^{+}, \frac{2 \pi}{\omega_{+}}\right)}$, then $\Sigma \gamma(\bar{z}, \bar{\tau}, \bar{p})<$ 0 , where $(\bar{z}, \bar{\tau}, \bar{p})$, in fact, take the form of either $\left(z^{*}, \tau_{j}^{+}, \frac{2 \pi}{\omega_{+}}\right)$or $\left(\bar{z}_{3}, \tau_{j}^{+}, \frac{2 \pi}{\omega_{+}}\right)$, $k=0,1,2, \cdots$. It follows together with [15, Theorem 3.3] that the connected component $\ell_{\left(z^{*}, \tau_{j}^{+}, \frac{2 \pi}{\omega_{+}}\right)}$through $\left(z^{*}, \tau_{j}^{+}, \frac{2 \pi}{\omega_{+}}\right)$in $\Sigma$ is unbounded. From (2.6) and (2.7), when $j>0$, we have $\frac{2 \pi}{\omega_{+}}<\tau_{j}^{+}$.

Now we prove that the projection of $\ell_{\left(z^{*}, \tau_{j}^{+}, \frac{2 \pi}{\omega_{+}}\right)}$onto $\tau$-space is $[\bar{\tau}, \infty)$, where $\bar{\tau} \leq \tau_{j}^{+}$. Clearly, it follows from the proof of Lemma 3.2 that system (1.1) with $\tau=0$ has no nontrivial periodic solutions. Hence, the projection of $\ell_{\left(z^{*}, \tau_{j}^{+}, \frac{2 \pi}{\omega_{+}}\right)}$onto $\tau$-space is away from zero.

For a contradiction, we suppose that the projection of $\ell_{\left(z^{*}, \tau_{j}^{+}, \frac{2 \pi}{\omega_{+}}\right)}$onto $\tau$-space is bounded. This means that the projection of $\ell_{\left(z^{*}, \tau_{j}^{+}, \frac{2 \pi}{\omega_{+}}\right)}$onto $\tau$-space is included in a interval $\left(0, \tau^{*}\right)$. Noticing $\frac{2 \pi}{\omega_{+}}<\tau_{j}^{+}$and applying Lemma 3.2, we have $0<p<\tau^{*}$, for $(z, \tau, p) \in \ell_{\left(z^{*}, \tau_{j}^{+}, \frac{2 \pi}{\omega_{+}}\right)}$. This implies that the projection of $\ell_{\left(z^{*}, \tau_{j}^{+}, \frac{2 \pi}{\omega_{+}}\right)}$onto $\tau$ space is bounded. Then, applying Lemma 3.1 we get that the connected component $\ell_{\left(z^{*}, \tau_{j}^{+}, \frac{2 \pi}{\omega_{+}}\right)}$is bounded. This contradiction completes the proof.

\section{Numerical simulations}

In this section, we investigate the following system:

$$
\left\{\begin{array}{l}
\dot{x}=x(t)\left[0.2-0.5 x(t-\tau)-\frac{5 y(t)}{1+0.5 x(t)}\right] \\
\dot{y}=y(t)\left[-0.5+\frac{5 x(t)}{1+0.5 x(t)}-0.5 y(t)\right] .
\end{array}\right.
$$


By the section 2 , we can obtain $E^{*}(0.1087,0.0307)$ and the following parameters: $\omega_{+}=0.3507, \omega_{-}=0.2867, \tau_{0}^{+}=4.5504, \tau_{0}^{-}=5.8492, \tau_{1}^{+}=22.4659, \tau_{1}^{-}=$ $27.7643, \tau_{2}^{+}=40.3813, \tau_{2}^{-}=49.6794, \tau_{3}^{+}=58.2967, \tau_{3}^{-}=71.5946, \cdots$. We choose $\tau=3, \tau=5.8492, \tau=27.7643, \tau=40.3813$, respectively, and $\left(x_{0}, y_{0}\right)$ is initiative value. Using Matlab Microsoft, we get the orbits of system (1.1) as Fig.4.1-4.4. From the figures, we can see that the previous theoretical analysis results are true.
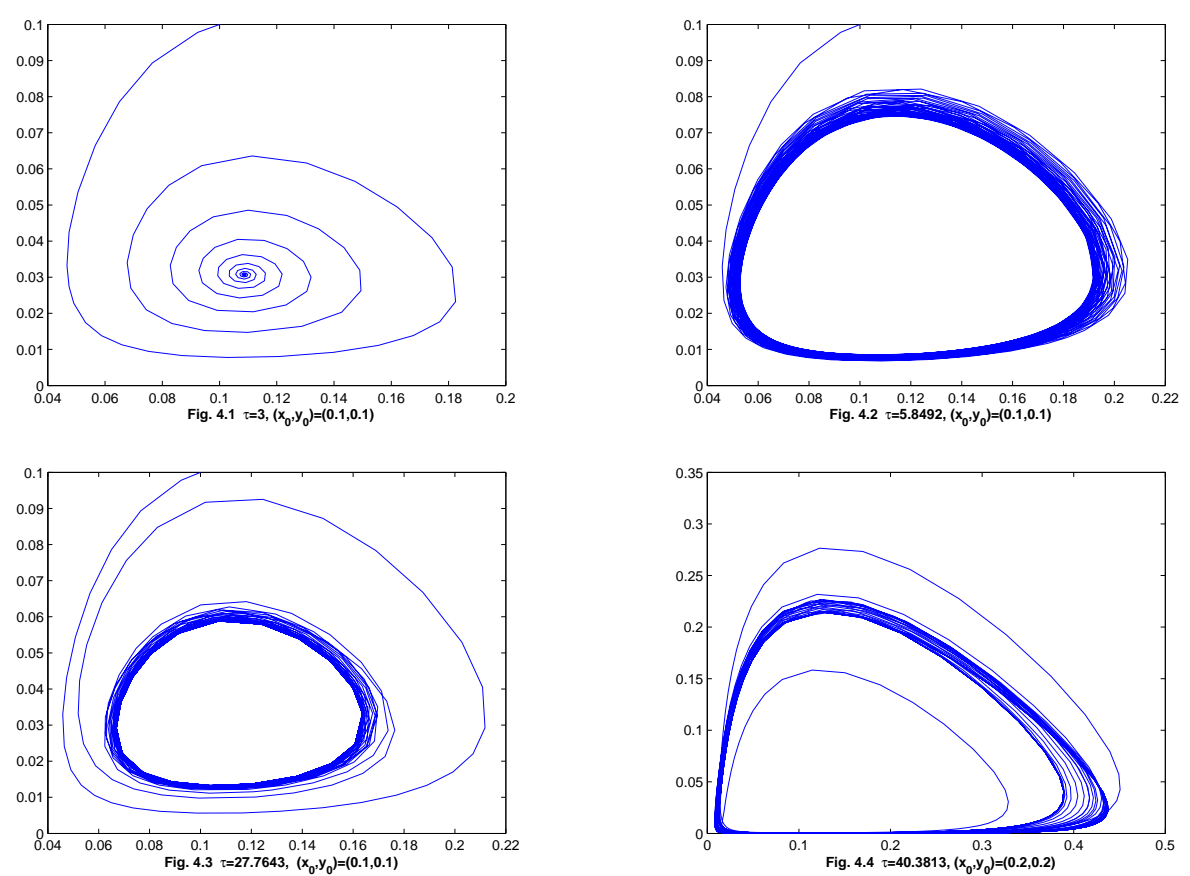

The Fig. 4.1 shows that $E^{*}$ is asymptotically stable with the initiative $\left(x_{0}, y_{0}\right)=$ $(0.1,0.1)$ when $\tau=3<\tau_{0}^{+}$. The Fig. 4.2 shows that $E^{*}$ is unstable and exists a stable periodic orbit with the initiative $\left(x_{0}, y_{0}\right)=(0.1,0.1)$ when $\tau=5.8492$. The Figs. 4.3 and 4.4 show that the periodic orbit still exists with the initiative $\left(x_{0}, y_{0}\right)=(0.1,0.1)$ and $\left(x_{0}, y_{0}\right)=(0.2,0.2)$ when $\tau=27.7643$ and $\tau=40.3813$, respectively.

\section{References}

[1] R. Nussbaum, Periodic solutions of some nonlinear autonomous functional equations, Ann. Mat. Pura Appl., 10(1974), 263-306. 
[2] M. Baptistiini, P. Tboas, On the existence and global bifurcation of periodic solutions to planar differential delay equations, J. Differential Equations, 127(1996), 391-425.

[3] J. Hale, S. Lunel, Introduction to Functional Differential Equations, in: Appl.Math. Sci., vol. 99, Spring- Verlag, New York, 1993.

[4] J. Wei, Q. Huang, Global existence of periodic solutions of linard equations with time delay, Dynam. Contin. Discrete Impuls. Systems Ser. A, 6(1999), 603-614.

[5] T. Zhao, Y. Kuang and H. Smith, Global existence of periodic solutions in a class of delayed Gause-type predatorCprey systems, Nonlinear Anal., 28(1997), 1373-1394.

[6] L. Erbe, K. Geba, W. Krawcewicz and J. Wu, $S^{1}$-degree and global Hopf bifurcations, J. Differential Equations, 98(1992), 277-298.

[7] W. Krawcewicz, J. Wu and H. Xia, Global Hopf bifurcation theory for considering fields and neural equations with applications to lossless transmission problems, Canad. Appl. Math. Quart., 1(1993), 167-219.

[8] W. Krawcewicz, J. Wu, Theory and application of Hopf bifurcations in symmetric functional differential equations, Nonlinear Anal., 35(1999), 845-870.

[9] S. Ruan, J.Wei, Periodic solutions of planar systems with two delays, Proc. Roy. Soc. Edinburgh Sect. A, 129(1999), 1017C1032.

[10] Y. Song, J.Wei and H. Xi, Stability and bifurcation in a neural network model with delay, Differential Equations Dynamic Systems, 9(2001), 321-339.

[11] Y. Song, J. Wei, Local and global Hopf bifurcation in a delayed hematopoiesis, Internat. J. Bifur. Chaos Appl. Sci. Engrg., 14(2004), 3909-3919.

[12] J. Wei, Y. Li, Hopf bifurcation analysis in a delayed Nicholson Blowflies equation, Nonlinear Anal., 60(2005), 1351-1367.

[13] J.Wei, Y. Li, Global existence of periodic solutions in a Tri-Neuron Network model with delays, 198(2004), 106-119.

[14] X. Wen, Z. Wang, The existence of periodic solutions for some models with delay, Nonlinear Anal. RWA, 3(2002), 567-581.

[15] J. Wu, Symmetric functional differential equations and neural networks with memory, Trans. Amer. Math. Soc., 35(1998), 4799-4838.

[16] S. Jung, On an asymptotic behavior of exponential functional equation, Acta Mathematica Sinica, English Series, 22(2006), 583-586.

[17] K. Jun, H. Kim, Stability problem for Jensen-type functional equations of cubic mappings, Acta Mathematica Sinica, English Series, 22(2006), 1781-1788.

[18] L. Hei, J. Wu, Existence and Stability of Positive Solutions for an Elliptic Cooperative System, Acta Mathematica Sinica, English Series, 21(2005), 1113-1120 .

[19] S. Ruan, Absolute stability, conditional stability and bifurcation in Kolmogorov-type predatorCprey systems with discrete delays, Quart. Appl. Math., 59(2001), 159-173.

[20] J. Wei, S. Ruan, Stability and bifurcation in a neural network model with two delays, Phys. D, 130(1999), 225-272 .

[21] S. Nakaoka, Y. Saito and Y. Takeuchi, Stability, delay, and chaotic behavior in a Lotka-Volterra predator-prey system, Mathematical Biosciences and Engineering, 3(2006), 173-187. 
[22] X. He, Stability and delays in a predatorCprey system, J. Math. Anal. Appl., 198(1996), 355-370.

[23] W. Wang, Z. Ma, Harmless delays for uniform persistence, J. Math. Anal. Appl., 158(1991), 256-268. 